(2) Open Access Full Text Article

\title{
Andrographolide promotes vincristine-induced SK-NEP-I tumor cell death via PI3K-AKT-p53 signaling pathway
}

This article was published in the following Dove Press journal:

Drug Design, Development and Therapy

28 September 2016

Number of times this article has been viewed

\author{
Mingsheng Zhang \\ Enda Xue \\ Wei Shao
}

Department of Pediatric Surgery, Liaocheng People's Hospital, Liaocheng, Shandong Province, People's Republic of China
Correspondence: Enda Xue Department of Pediatric Surgery, Liaocheng People's Hospital, 67 Dongchang Road, Liaocheng 252000, Shandong Province, People's Republic of China Email enda_xue@hotmail.com
Background: Nephroblastoma (Wilms' tumor [WT]) is the most common malignant renal cancer in children. Although the outcome of WT has significantly improved as a result of the combination of surgery, chemotherapy, and radiotherapy; in some cases WT results in severe complications. Thus, novel strategies that would decrease treatment burden are required. The aim of the current study was to investigate the synergistic antitumor effect of andrographolide (AND) in combination with vincristine (VCR) on WT cells.

Methods: Cell Counting Kit- 8 assay was used to investigate the synergistic antiproliferation effect of AND and/or VCR on SK-NEP-1 cells in vitro. Meanwhile, SK-NEP-1 xenografts were used to detect the antitumor effect in vivo. Apoptosis and autophagy were then detected by Annexin V, monodansylcadaverine staining. Finally, the underlying signaling transduction was determined with Western blotting.

Results: The combination of AND with VCR significantly suppressed SK-NEP-1 cell proliferation in vitro and inhibited xenograft tumor growth in vivo, compared with AND or VCR treatment alone. In addition, the synergistic antitumor effect of AND on the cells was due to an increased apoptosis, not autophagy. Moreover, PI3K-AKT-p53 signaling pathway was involved in the process of combination treatment, which was confirmed when a selective AKT activator was applied.

Conclusion: The combination of AND with VCR has a strong synergistic antitumor effect on WT via PI3K-AKT-p53 signaling pathway, thereby representing a potential treatment for WT in the near future.

Keywords: andrographolide, vincristine, p53, drug combination

\section{Introduction}

Nephroblastoma is known as a Wilms' tumor (WT) after Dr Max Wilms who first described it in 1899. WT is the most common renal malignancy in childhood that typically occurs in children aged 3-4 years. As a result of the collaborative efforts of surgeons, pediatricians, pathologists, and radiation oncologists, the outcome of WT has improved significantly over the past few decades: the 5-year survival rate has improved from $20 \%$ in the 1960 s to $>85 \%$ in the current decade. The National Wilms' Tumor Study Group and the International Society of Pediatric Oncology conducted sequential studies of treatment for children with WT. ${ }^{1-4}$ However, in many developing countries, the outcome of treatment is still poor due to factors such as late presentation, persisting poverty, the possibility of a more aggressive tumor, and lack of collaboration among institutions. ${ }^{5,6}$ 
Currently, two distinct types of histopathologies are available based on prognosis favorable $(>90 \%)$ and unfavorable $(6 \%-10 \%)$. Anaplasia, which is associated with unfavorable histology, may be present in $\sim 5 \%$ of the WTs and can be characterized as focal or diffuse. ${ }^{7}$ Current management emphasizes reducing the morbidity of treatment for low-risk patients and reserving more intensive treatment for selected high-risk patients for whom outcome remains poor. Although patients with diffuse anaplastic histology received much more intensive treatment, including vincristine (VCR), doxorubicin, cyclophosphamide, and etoposide for 24 weeks, the outcome was still poor. ${ }^{8,9}$ Therefore, there is an urgent need for the development of a new agent or new combinations to decrease the treatment intensity and recurrence rate of the unfavorable patients.

Currently, herbal extracts are widely used as a complimentary treatment for various diseases. Andrographolide (AND) is the most active component that is isolated from the extract of Andrographis paniculata. For many years, AND had been used in traditional Asian medicine in the treatment of upper respiratory, fever, diarrhea, rheumatoid arthritis, and so on, and recently, it was found that AND possesses various biological activities, including anti-inflammatory, immune modulatory, antitumor effects, and so on. ${ }^{10}$ Also, it was found that AND inhibited tumor growth in cancer cell lines by inducing cell cycle arrest or apoptosis. ${ }^{11}$ However, till date, no studies had been reported on the antitumor effects of AND on WT. In the current study, in order to develop a new treatment for unfavorable WT, for the first time, AND was combined with VCR, a well-known vinca alkaloid anticancer drug for WT, to test their effects on SK-NEP-1 kidney tumor cells. The aim of current study was to investigate the synergistic antitumor effect of AND in combination with VCR on WT cells. Many biochemical methods were used to determine the anticarcinoma behaviors of AND plus VCR, and then the possible underlying mechanisms were investigated further.

\section{Materials and methods}

\section{Reagents}

The antibodies AKT, phospho-AKT (p-AKT), p53, phosphop53 (p-p53), ERK, phospho-ERK (p-ERK), and $\beta$-actin were purchased from Cell Signaling Technology (Beverly, MA, USA). VCR (purity, 98\%) was purchased from Guangzhou Huanye Pharmaceutical Co., Ltd. (Guangzhou, People's Republic of China). The AND ( $\geq 98 \%$ purity) and AKT activator SC-79 were purchased from Sigma-Aldrich (St. Louis, MO, USA). All reagents were of analytical grade and were used without further purification.

\section{Cell culture and treatment}

SK-NEP-1 human kidney (WT) cell line was obtained from the American Type Culture Collection (Rockville, MD, USA). It was kept in the Maccyo'5 medium (Thermo Fisher Scientific, Waltham, MA, USA) supplemented with $15 \%$ heat-inactivated fetal bovine serum (Thermo Fisher Scientific) in a humidified incubator with $5 \% \mathrm{CO}_{2}$ at $37^{\circ} \mathrm{C}$. VCR and AND were dissolved in dimethyl sulfoxide (DMSO). Cells were treated for different purposes and were divided into five treatment groups: 1) treated with increasing concentrations of either a single drug (3-15 nM of VCR or 5-25 $\mu \mathrm{M}$ of AND); 2) treated with half maximal inhibitory concentration $\left(\mathrm{IC}_{50}\right)$ of $\mathrm{VCR}$ in combination with AND at different concentrations of 10,20 , and $25 \mu \mathrm{M} ; 3$ ) treated with increasing concentrations of VCR in combination with AND at different concentrations of 10, 20, and $25 \mu \mathrm{M}$; 4) $10.8 \mathrm{nM}$ VCR, pretreated with or without $1 \mu \mathrm{M}$ SC-79 for 6 hours; 5) treated with $10.8 \mathrm{nM} \mathrm{VCR}+25 \mu \mathrm{M}$ AND, pretreated with or without $1 \mu \mathrm{M}$ SC-79 for 6 hours. DMSO treatment group was set as a control. Then, cell morphology was determined by examining the culture cell without preliminary fixation under inverted microscope at a magnification of $\times 100$. The micrographs were recorded using a Nikon phase contrast microscope (Nikon, Tokyo, Japan).

\section{Detection of cell proliferation by using CCK-8 assay}

Cell proliferation was assessed by using the Cell Counting Kit-8 (CCK-8; Dojindo Laboratories, Kumamoto, Japan). Cells were seeded into 96 -well plates $(100 \mu \mathrm{L} /$ well; 5,000 cells/well), and four wells were included in each group. After 6 hours of incubation at $37^{\circ} \mathrm{C}$ in an environment with $5 \% \mathrm{CO}_{2}$, cells were treated with or without test samples as required. After incubation for 24,48 , and 72 hours, $10 \mu \mathrm{L}$ of CCK-8 solution was added to each well, which was followed by an additional 3 hours of incubation. Optical density was determined on a spectrophotometer (Microplate Reader 550; Bio-Rad Laboratories, Hercules, CA, USA) at a wavelength of $450 \mathrm{~nm}$. The $\mathrm{IC}_{50}$ values of VCR solution or of VCR plus AND combination were calculated on the basis of a series of dose-response data using GraphPad Prism software, version 6 (GraphPad, La Jolla, CA, USA). Drug combination analyses were performed using CompuSyn (ComboSyn, Inc., New York, NY, USA). The CompuSyn software generates the combination index $(\mathrm{CI})$ value for a particular combination of VCR with AND based on the data from the CCK-8 assay, whereby additivity, synergy, and antagonism were reflected by $\mathrm{CI}$ values of $0.9-1.1,<0.9$, and $>1.1$, respectively. ${ }^{12}$ 


\section{Apoptosis assay}

Cellular apoptosis assay was performed according to the manufactory of R\&D TACS ${ }^{\circledR}$ Annexin V-FITC (fluorescein isothiocyanate) kit. After treatment with or without test samples, cells were washed twice with cold phosphate-buffered solution (PBS) and then resuspended in $1 \times$ binding buffer at the concentration of approximately $1 \times 10^{6}$ cells $/ \mathrm{mL}$; then, $5 \mu \mathrm{L}$ of Annexin $\mathrm{V}$ and propidium iodide (PI) solution was added to every $100 \mu \mathrm{L}$ of the cell suspension (approximately $1 \times 10^{5}$ cells). The cells were gently mixed and incubated at room temperature in a dark environment for 15 minutes; then, $400 \mu \mathrm{L}$ of $1 \times$ binding buffer was added to each tube. Within 1 hour, the cells were analyzed by flow cytometry on FACScan (Becton Dickinson, Bergen County, NJ, USA).

\section{Caspase- 3 activity assay}

Caspase-3 activity was assayed in cellular extracts using the Caspase-3 Colorimetric Assay kit (R\&D Systems, Minneapolis, MN, USA). The cells were cultured and treated as Group 2 for 48 hours and then harvested and washed twice with cold PBS. Then, the cell pellets were lysed with $50 \mu \mathrm{L}$ of cold lysis buffer, followed by 1 hour of incubation on ice. The supernatant was collected and added with $50 \mu \mathrm{L}$ of reaction buffer, followed by $5 \mu \mathrm{L}$ of caspase- 3 substrate. The suspension was collected and transferred to a 96-well micro-titer plate and incubated at $37^{\circ} \mathrm{C}$ for 2 hours before measurement at $405 \mathrm{~nm}$ using an enzyme-linked immunosorbent assay micro plate reader (Bio-Rad).

\section{Detection of autophagic vacuoles with monodansylcadaverine}

Monodansylcadaverine (MDC) staining has been widely reported to label autophagic vacuoles in vitro. ${ }^{13}$ The cells were cultured in 24-well plates and treated as Group 2 for 48 hours. Then, the cells were incubated with $0.05 \mathrm{mM}$ MDC (Sigma-Aldrich) for 30 minutes at $37^{\circ} \mathrm{C}$. These cells were washed with PBS three times and analyzed using a fluorescence microscope (Eclipse TE2000U; Nikon) equipped with a filter system. The graphs represent quantification of three independent experiments, and a total of 200 cells were counted per treatment.

\section{Western blotting}

Expression levels of the cellular proteins were determined using Western blotting assays. Cells were washed with cold PBS and lysed with Radioimmunoprecipitation assay (RIPA), lysis buffer for cells (1\% Triton X-100, 0.1\% sodium dodecyl sulfate (SDS), 1\% deoxycholate (DOC),
$10 \mathrm{mM}$ tris(hydroxymethyl)aminomethane- $\mathrm{HCl} \mathrm{pH} 7.4$, $150 \mathrm{mM} \mathrm{NaCl}, 5 \mathrm{mM}$ ethylenediaminetetraacetic acid, $10 \mu \mathrm{g} / \mathrm{mL}$ leupeptin, and $1 \mathrm{mM} \mathrm{Na} \mathrm{VO}_{4}$ ), followed by centrifugation at $4{ }^{\circ} \mathrm{C}$ for 30 minutes. Protein concentration was determined using the bicinchoninic acid (BCA) Protein Assay Kit (Promega, Madison, WI, USA). The supernatants with equal amount $(50 \mu \mathrm{g})$ of protein sample in loading buffer were loaded on 10\%-12\% SDS-polyacrylamide gel electrophoresis (PAGE) and transferred to polyvinylidene fluoride (PVDF) (Bio-Rad). The membranes were blocked with 5\% (weight/volume) nonfat milk in Tris-buffered saline-Tween and probed with the primary antibodies (1:1,000 dilution) overnight at $4^{\circ} \mathrm{C}$. After three washes with Tris-buffered saline-Tween, the blots were incubated with appropriately diluted horseradish peroxidase-conjugated secondary antibodies (1:2,000 dilution) for 2 hours. After three 15 minutes of Tris-buffered saline-Tween washing steps, the immunoreactive protein bands were visualized by the Enhanced Chemiluminescence Kit (GE Healthcare, Buckinghamshire, UK). Protein bands were visualized after the exposure of the membrane to Kodak X-ray Film.

\section{Tumor model}

BALB/c-nude mice, 6-8 weeks old, were used for the establishment of SK-NEP-1 xenografts model. The mice were provided by Vital River (Beijing, People's Republic of China) and housed in barrier facilities on a 12-hour light/dark cycle. Then, $3 \times 10^{6}$ SK-NEP-1 cells were subcutaneously injected in the right flank of the mice. Tumor size was monitored once a week, and tumor volumes were calculated by using the following formula: tumor size $=$ length $\times$ width $^{2} \times 0.5$. When the tumor size reached $\sim 150 \mathrm{~mm}^{3}$ ( 1 week after cell implantation), the mice were randomized to four groups and received vehicle (normal saline, oral, once daily), $100 \mathrm{mg} / \mathrm{kg}$ AND (oral, once daily), $0.2 \mathrm{mg} / \mathrm{kg}$ VCR (intraperitoneal, once weekly), or AND plus VCR for 4 weeks. The AND and VCR were dissolved in normal saline, which were freshly prepared before the treatment. The mice were sacrificed, and the tumors were weighted after 4 weeks of treatment. This study was reviewed and approved by Liaocheng People's Hospital Animal Center Committee. All experiments were performed following Regulations for the Administration of Affairs Concerning Experimental Animals.

\section{Statistical analysis}

All data and results presented in this study are representative of, or calculated from, at least three independent experiments. All statistical analyses were performed using the Statistical 
Product and Service Solutions software (SPSS; Version 13.0; SPSS Inc., Chicago, IL, USA). Data were analyzed by Student's $t$-test. $P$-values $<0.05$ were statistically significant. Values are represented as the mean \pm standard deviation.

\section{Results}

\section{Combination treatment of VCR with} AND had a synergistic antiproliferative effect on SK-NEP-I cells

First, we used CCK-8 assay to assess whether the combination of VCR with AND had a synergistic antiproliferative effect on SK-NEP-1 cells. The cells were treated with increasing concentrations of either drug alone or in combination, as indicated in the "Methods" section (Treatment Groups 1, 2, and 3). Figure $1 \mathrm{~A}$ shows that VCR decreased the SK-NEP-1 cell viability in a dose- and time-dependent manner, and the $\mathrm{IC}_{50}$ value at 48 hours is $10.8 \mathrm{nM}$. However, the antiproliferative effect of AND was very weak, although $25 \mu \mathrm{M}$ AND treatment for 72 hours had $<20 \%$ inhibition (Figure $1 \mathrm{~B}$ ). As shown in Figure $1 \mathrm{~A}$ and $\mathrm{C}$ and Table 1, the $\mathrm{IC}_{50}$ values decreased when treated with both drugs than with either drug alone. The CI values at $\mathrm{IC}_{50}$ were $<0.9$ when $\mathrm{AND}$ was set at 10,20 , and $25 \mu \mathrm{M}$. These results indicated that higher concentrations of AND had a synergistic interaction with VCR. In addition, the cells were examined under a inverted microscope after treatment. The control group showed normal cellular morphology and density. Nevertheless, changed cellular morphology (with cells becoming round and shrunken) and a decreased cell number were observed upon drug treatment, especially in the VCR plus AND combination treatment group (Figure 1D). This result was consistent with the results of CCK-8 assay.

\section{Caspase-3-dependent apoptosis was involved in VCR- or/and AND-induced SK-NEP-I cell death}

We next determined whether VCR plus AND-induced SK-NEP-1 cell death via apoptosis using Annexin V/PI staining. The experimental concentrations of drugs were chosen according to CCK-8 assay (Treatment Group 2). The Annexin V+PI-cells (lower right quadrant) represented early apoptotic cells, and the Annexin V + PI+ cells (upper right quadrant) represented advanced apoptotic cells and/or necrotic cells. As shown in Figure 2A and B, control group did not show any significant apoptosis, and the percentage of early apoptotic and late apoptotic cells was only 5\%. After treatment with $10.8 \mathrm{nM}$ VCR for 48 hours, the corresponding quantity was $32 \%$. Moreover, an addition of AND with $10.8 \mathrm{nM} \mathrm{VCR}$ dramatically increased the apoptotic rate of SK-NEP-1 cells, and the corresponding values for 10,20 , and $25 \mu \mathrm{M}$ AND
A

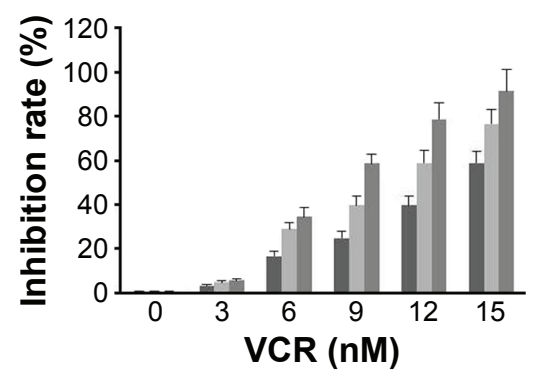

B

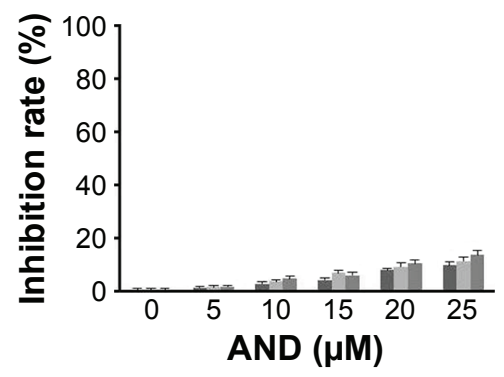

C

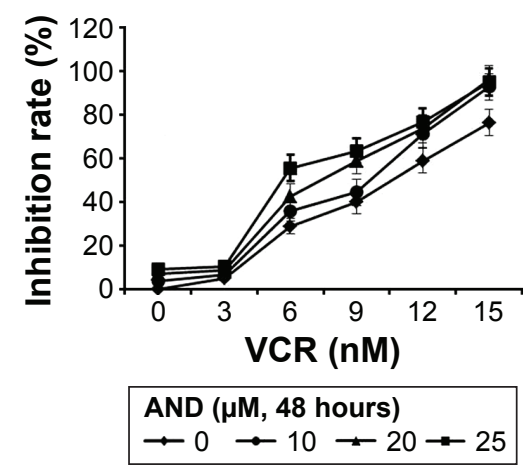

$\rightarrow 0 \rightarrow 10 \rightarrow 20 \rightarrow 25$

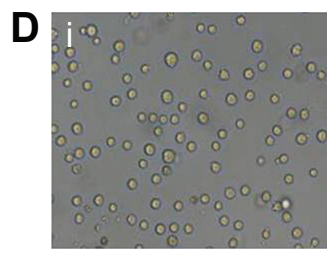

Control

24 hours 48 hours 72 hours

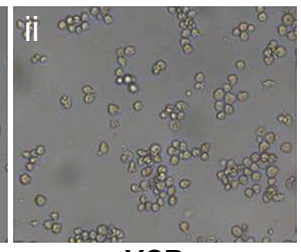

VCR

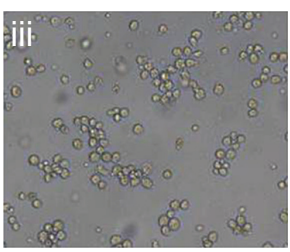

VCR +10

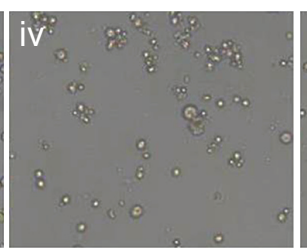

VCR +20

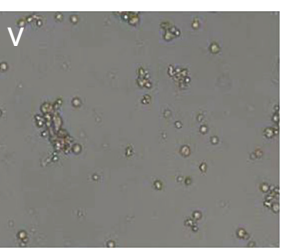

VCR +25

AND $(\mu \mathrm{M})$

Figure I Growth inhibition effect of SK-NEP-I cells treated with VCR, AND, and VCR plus AND.

Notes: (A and B) SK-NEP-I cells were treated with empty vehicle, VCR (0-15 nM), or AND (0-25 $\mu$ M) for different time periods, and then, cell inhibition rate was determined by CCK-8 assay. The data were obtained from an average of four independent experiments. (C) SK-NEP-I cells were treated with the combination of VCR with AND for 48 hours. (D) Representative microscopy images captured by a Nikon phase contrast microscope (Nikon, Tokyo, Japan) showing the effect of cells treated with vehicle (i); $10.8 \mathrm{nM}$ VCR (ii); $10.8 \mathrm{nM}$ VCR + $10 \mu \mathrm{M}$ AND (iii); $10.8 \mathrm{nM}$ VCR +20 $\mu$ M AND (iv); or $10.8 \mathrm{nM}$ VCR +25 $\mu$ M AND (v) for 48 hours (magnification $\times 100$ ). Abbreviations: AND, andrographolide; CCK-8, Cell Counting Kit-8; VCR, vincristine. 
Table I The $\mathrm{IC}_{50}$ and $\mathrm{Cl}$ values of VCR alone or VCR plus AND combination treatment against SK-NEP-I cells

\begin{tabular}{lll}
\hline Drugs & $\begin{array}{l}\text { IC }_{50} \text { values } \\
(\mathbf{n M}, \mathbf{4 8} \text { hours })\end{array}$ & $\begin{array}{l}\text { Cl values } \\
\text { at IC }\end{array}$ \\
\hline VCR & 10.8 & NA \\
VCR $+10 \mu M$ AND & 9.5 & 0.98 \\
VCR $+20 \mu M$ AND & $6.6^{*}$ & 0.66 \\
VCR $+25 \mu M$ AND & $5 . I^{*}$ & 0.58 \\
\hline
\end{tabular}

Notes: The concentration of $\mathrm{VCR}$ ranged from 0 to $15 \mathrm{nM}$. $* P<0.05$, compared to VCR alone treatment.

Abbreviations: AND, andrographolide; $\mathrm{Cl}$, combination index; $\mathrm{IC}_{50}$, half maximal inhibitory concentration; NA, not applicable; VCR, vincristine.

were $39 \%, 50 \%$, and $56 \%$, respectively $(P<0.05)$. Therefore, VCR-induced SK-NEP-1 cell apoptosis, which was significantly enhanced by AND in a dose-dependent manner.

It is known that caspase-3 is characterized as both a marker and an ultimate executioner of cell apoptosis. ${ }^{14}$ To confirm the apoptotic effect, we evaluated the activity of caspase-3 in SK-NEP-1 cells. It was found that the combination of VCR with 20 or $25 \mu \mathrm{M}$ AND induced a significant increase of caspase-3 cleavage compared with VCR alone treatment (Figure $2 \mathrm{C} ; P<0.01$ ). This result was consistent with the results of Annexin V/PI staining.

\section{Autophagy was not involved in VCR- or/ and AND-induced SK-NEP-I cell death}

Autophagy plays an important role in apoptosis of various kinds of cancer cell lines. ${ }^{15}$ In order to determine whether it plays a similar role in SK-NEP-1 cells, in the current research, MDC staining assay was used. The images under the fluorescence microscopy revealed that no matter whether it was treated with VCR alone or VCR plus AND combination, the percentage of autophagic vacuoles showed no difference compared with control group (Figure 3A). In the normalized data, no more than $5 \%$ of cells were MDC positive (Figure 3B). This result demonstrated that autophagy was not involved in VCR- or/and AND-induced SK-NEP-1 cell death.

\section{The combination treatment of VCR with AND upregulated $\mathrm{p}-\mathrm{AKT}$ and $\mathrm{p}-\mathrm{p} 53$ levels}

The transcription factor p53 has been shown to induce apoptosis by causing mitochondrial dysfunction. It is reported that an increased expression of p-p53 was found in AND-induced apoptosis in various cancer cell lines. ${ }^{16-18}$ Then, to explore the role of p53 in VCR- or/and AND-induced SK-NEP-1 cell apoptosis, protein expression changes were examined by using Western blotting. SK-NEP-1 cells were treated as Group 2 for
48 hours. Western blotting analyses revealed an increase in p-p53 level and a decrease in p-AKT level in the cells treated with VCR/AND combination compared with control or VCR alone, and p-ERK was not affected (Figure 4A). In the normalized data (Figure 4B), p-p53 protein reached its highest level at $20 \mu \mathrm{M}$ AND treatment. These data illustrated that PI3KAKT-p53 signal pathway was involved in the combination treatment of VCR and AND in SK-NEP-1 cells.

\section{AKT activator partly reversed apoptosis induced by VCR plus AND combination treatment in SK-NEP-I cells}

In order to further confirm whether PI3K-AKT-p53 pathway was involved in AND- or/and VCR-induced apoptosis, cells with $1 \mu \mathrm{M}$ SC-79, an activator of AKT, ${ }^{19}$ were pretreated prior to the test samples (Treatment Group 4). The results of CCK-8 indicated that cell growth inhibition rate caused by VCR plus AND combination treatment was partly reversed by $\mathrm{SC}-79$ pretreatment (Figure $5 \mathrm{~A} ; P<0.05$ ). Data from Western blotting (Figure 5B and C) indicated that SC-79 restored the protein expression change of p-AKT and p-p53 caused by VCR plus AND combination treatment. On the other hand, when cells were treated with VCR alone, SC-79 pretreatment could not reduce the inhibition rate (Figure 5A), which indicated that cell death induced by VCR alone was not through AKT pathway. Thus, from all these data, it was concluded that AND had an antiapoptosis effect on SK-NEP-1 cell through PI3K-AKT-p53 signal pathway when combined with VCR.

\section{AND plus VCR significantly inhibited SK- NEP-I xenograft tumor growth in vivo}

In order to investigate whether AND and VCR have a synergetic antitumor effect on WT in vivo, SK-NEP-1 cells were injected into nude mice. The mice were sacrificed after 4 weeks of dosing. The results indicated that $100 \mathrm{mg} / \mathrm{kg}$ AND had a very limited antitumor effect, and VCR inhibited tumor growth on xenografts significantly. Similar to the in vitro results, the combination of AND with VCR had a dramatically synergetic antitumor effect on SK-NEP-1 xenografts compared with VCR group (Figure 6A-C). In addition, the results also showed that no obvious changes in body weight were observed between the treated groups and vehicle group (Figure 6D).

\section{Discussion}

The excellent outcome of WT has been achieved during the past few decades; however, scientists are still seeking new 

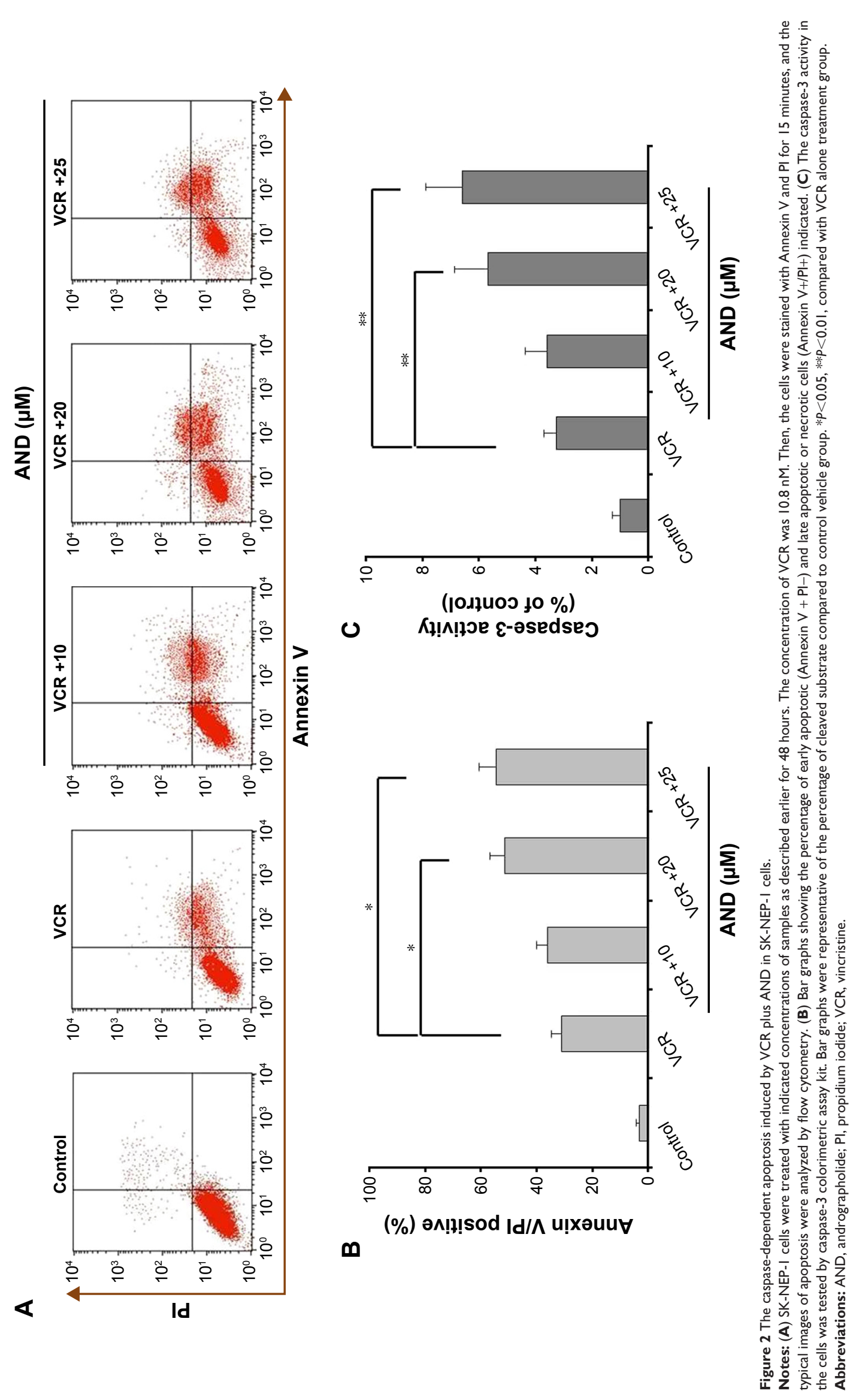


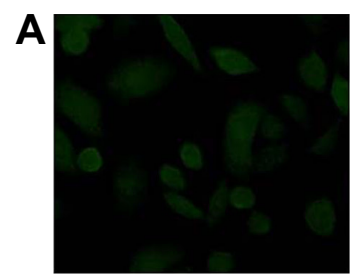

Control

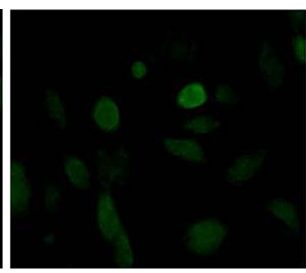

VCR

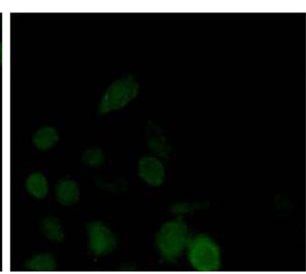

VCR +10

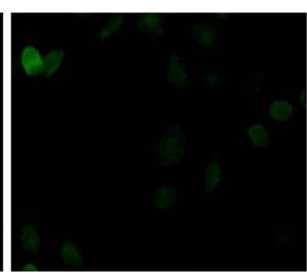

VCR +20

AND $(\mu \mathrm{M})$

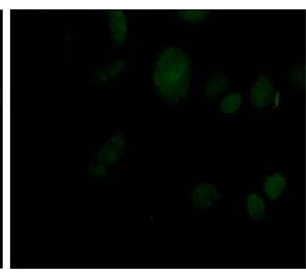

VCR +25

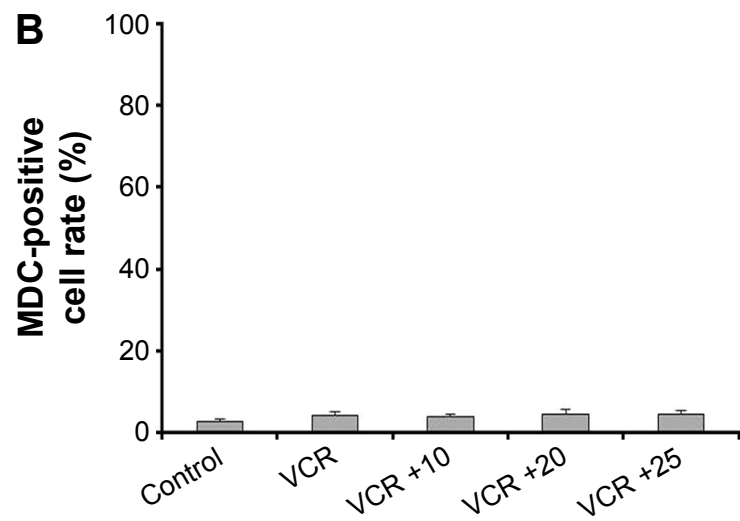

Figure 3 Cell growth inhibition induced by AND plus VCR was not dependent on autophagy.

Notes: (A) SK-NEP-I cells were treated with empty vehicle, $10.8 \mathrm{nM}$ VCR only, and in combination with I0, 20, and $25 \mu \mathrm{M}$ AND for 48 hours. Representative fluorescence microscopy images of cells stained with MDC. Green circles are representative of autophagic vacuoles. (B) Quantification of the percentage of MDC staining-positive cells. Abbreviations: AND, andrographolide; MDC, monodansylcadaverine; VCR, vincristine.

drugs and new combinations of treatment for patients with high-risk WT, including those with anaplastic, bilateral, and recurrent favorable histology tumors. Yan et al had focused on the histone acetylation target of WT and found that LBH589 caused apoptotic cell death in SK-NEP-1 cells. ${ }^{20}$ Tao et al found that YM155, a novel small-molecule survivin suppressant, could inhibit SK-NEP-1 tumor growth in vitro and in vivo. ${ }^{21}$ In recent decades, AND has been considered to be a potent anticancer plant extract. Rajagopal et al tested the anticarcinogenic activity of AND upon numerous cancer cell lines. AND inhibited the growth of many types of cancer cells by $50 \%\left(\mathrm{IC}_{50}\right)$ at a concentration range of $5-15 \mu \mathrm{M} .^{22}$
A

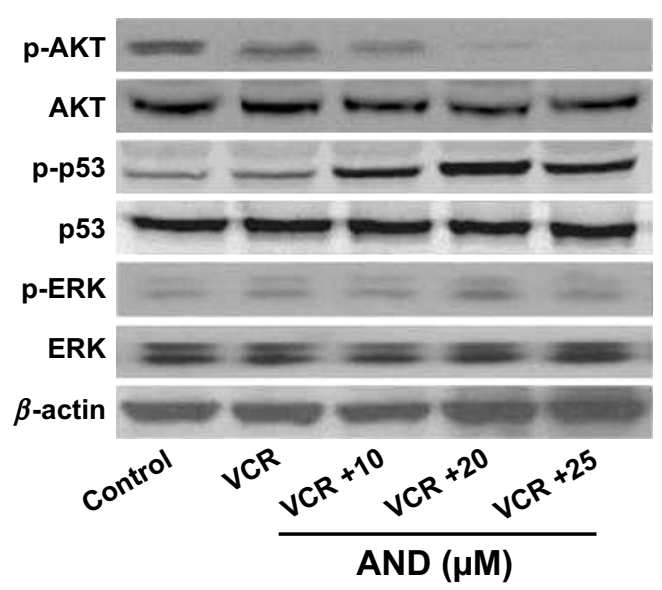

B

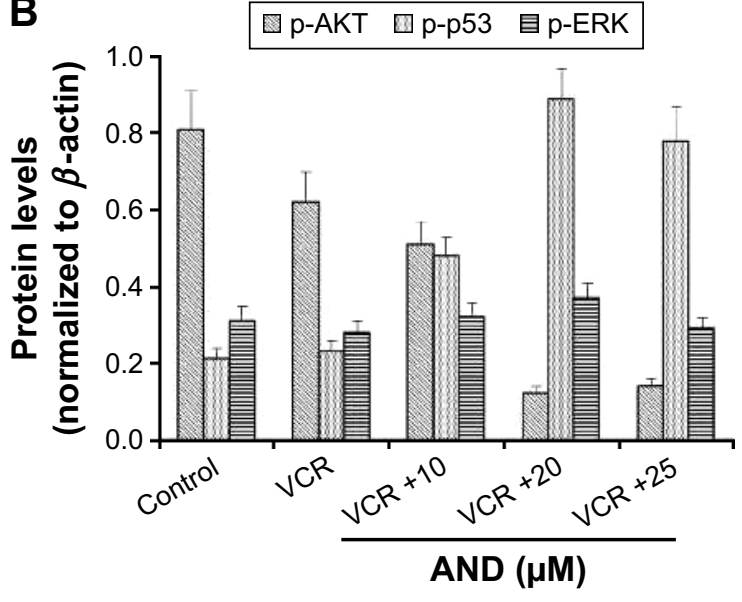

Figure 4 P-p53 and p-AKT protein levels were upregulated by VCR plus AND treatment in SK-NEP-I cells.

Notes: (A) Western blotting analysis of total AKT, p53, ERK and p-AKT, p-p53, and p-ERK activity in SK-NEP-I cells treated with I0.8 nM VCR alone or in combination with 10, 20, and $25 \mu \mathrm{M}$ AND for 48 hours. (B) Bar graphs showing the quantification of the $\mathrm{p}$-AKT, $\mathrm{p}$-p53, and $\mathrm{p}$-ERK activity in the cells. Data were normalized to $\beta$-actin. Abbreviations: AND, andrographolide; VCR, vincristine. 


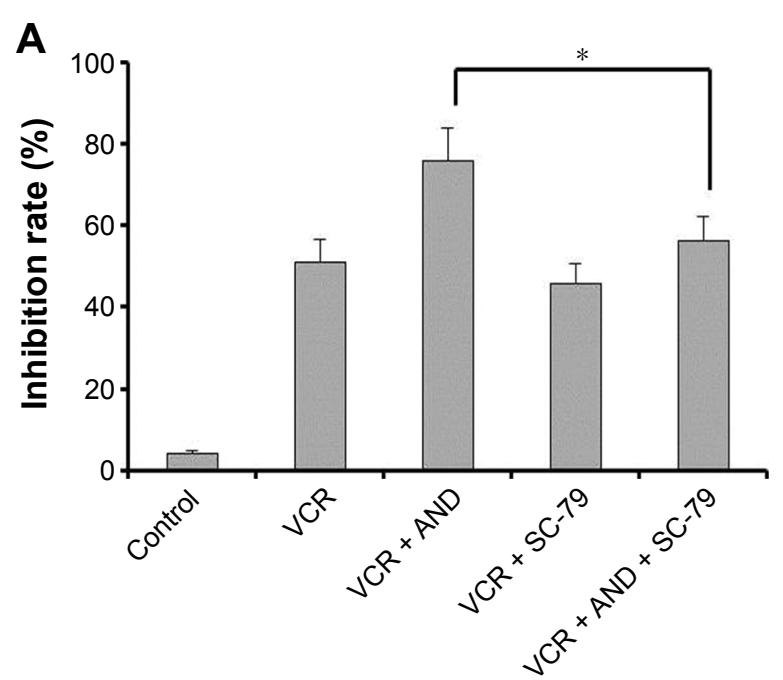

\section{B}
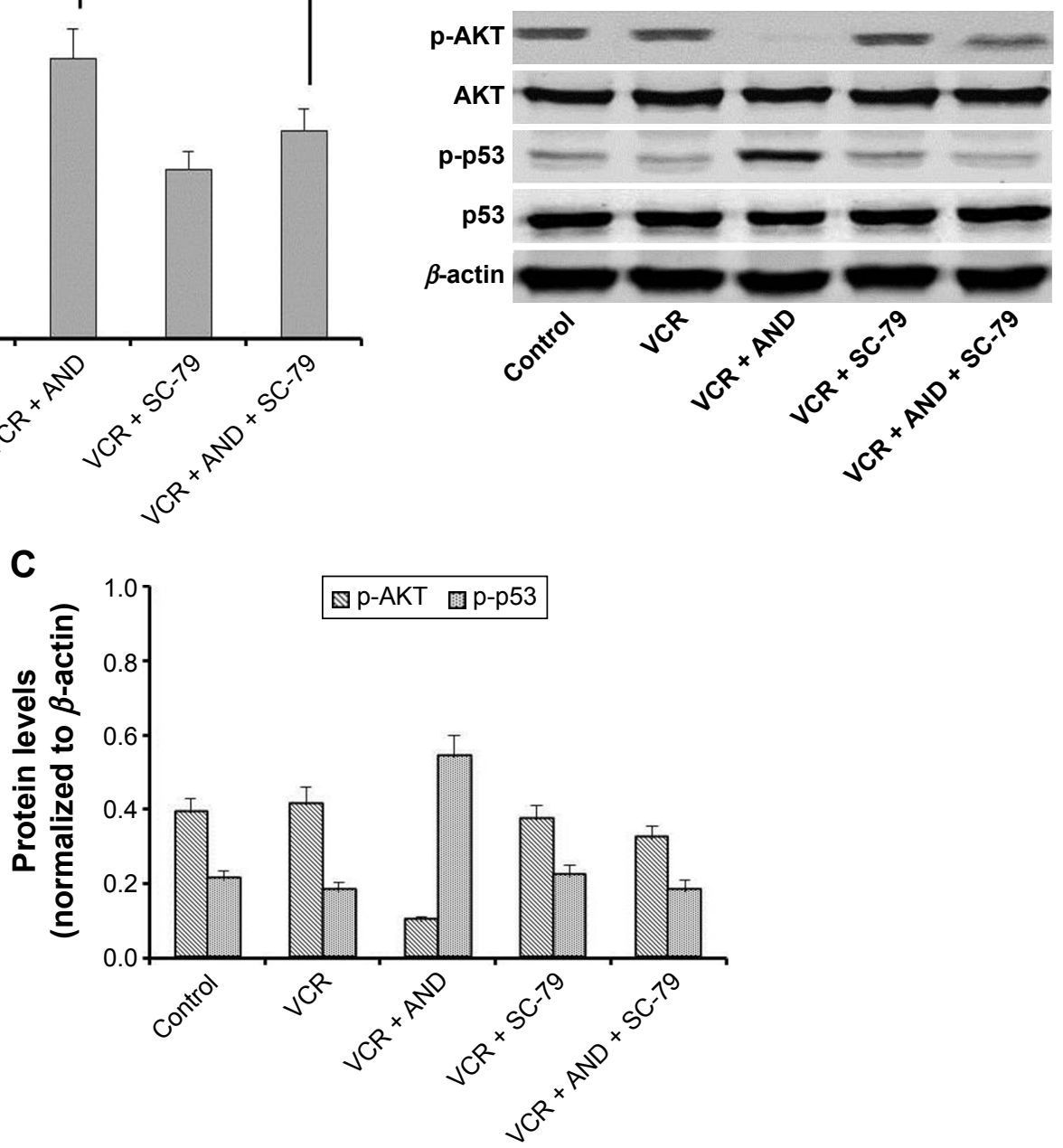

Figure 5 AKT activator modulates apoptotic cell death and protein expression levels induced by VCR plus AND treatment in SK-NEP-I cells.

Notes: (A) SK-NEP-I cells were pretreated with or without SC-79 for 6 hours and then treated with VCR, VCR + AND, VCR + SC-79, or VCR + AND + SC-79 for 48 hours. Cell growth inhibition rate was significantly reduced by SC-79 pretreatment. (B) Western blotting analysis of p-AKT and p-p53 protein level changes in the cells. (C) Bar graphs showing the quantification of the $\mathrm{p}$-AKT and $\mathrm{p}$-p53 expression levels in the cells. Data were normalized to $\beta$-actin. $* \mathrm{P}<0.05$, compared to VCR plus AND treatment group.

Abbreviations: AND, andrographolide; VCR, vincristine.

In the current study, the anticancer mechanisms of AND combined with VCR were investigated in order to test its effects on WT tumor in vitro and in vivo. In addition, this is the first time AND being combined with VCR for the treatment of WT.

Data from CCK-8 assay showed that AND alone (concentration, 5-25 $\mu \mathrm{M}$ ) had no significant inhibitory effect on the viability of SK-NEP-1 cells. This result was different from the results presented earlier, and AND was potent in many cancer cell lines. ${ }^{22}$ When VCR was combined with AND, a significant decrease of cell viability was observed in SK-NEP-1 cells compared with VCR or AND treatment alone. These results were consistent with the results observed under the microscopy and further verified by in vivo study.
The data suggested that AND may be potentially useful as a biochemical modulator to enhance the therapeutic effects of VCR in WT chemotherapy. Increased sensitivity of SKNEP-1 cells to VCR was further confirmed by Annexin-5/PI in the presence of AND. AND/VCR combination treatment group showed a higher proportion of Annexin V/PI double staining, compared with VCR alone group, and it was in a caspase-3-dependent manner, which was similar to the results of the studies of Yan et al and Tao et al. ${ }^{20,21}$

It is known that VCR is an antitumor drug that inhibits microtubule polymerization, causes $\mathrm{G} 2 / \mathrm{M}$ arrest, and induces apoptosis. Previous studies had reported that ANDinduced apoptotic cell death was via different molecular pathways, such as activation of proapoptotic JNK 
A

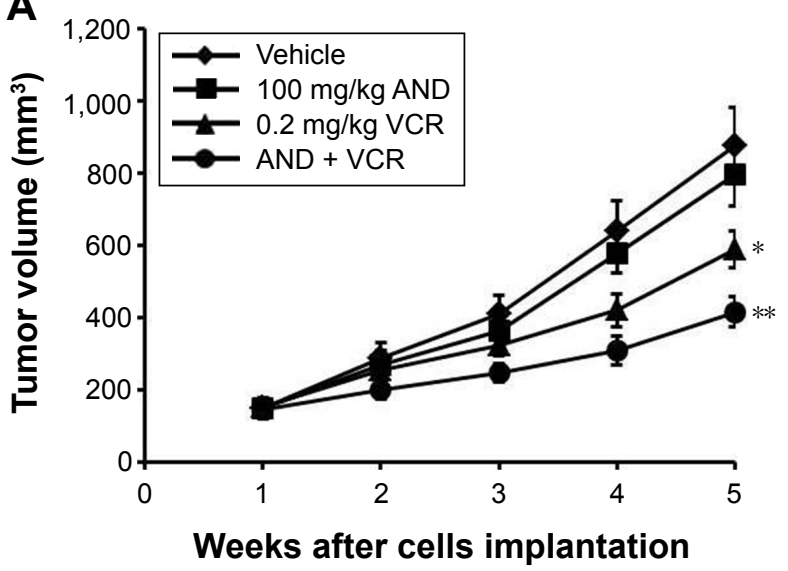

B

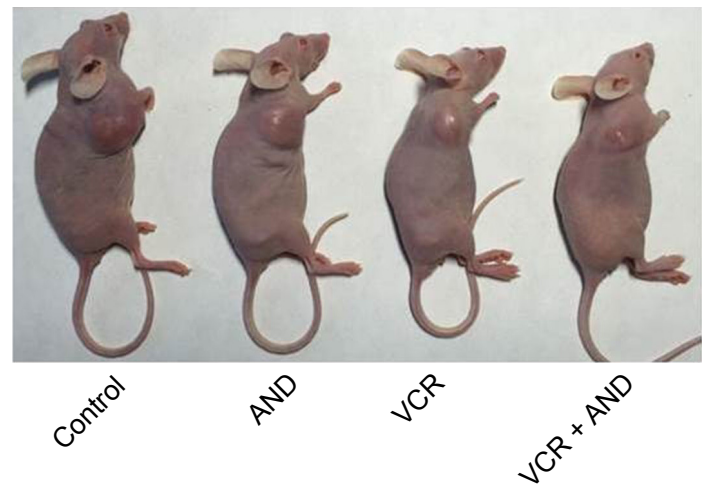

C

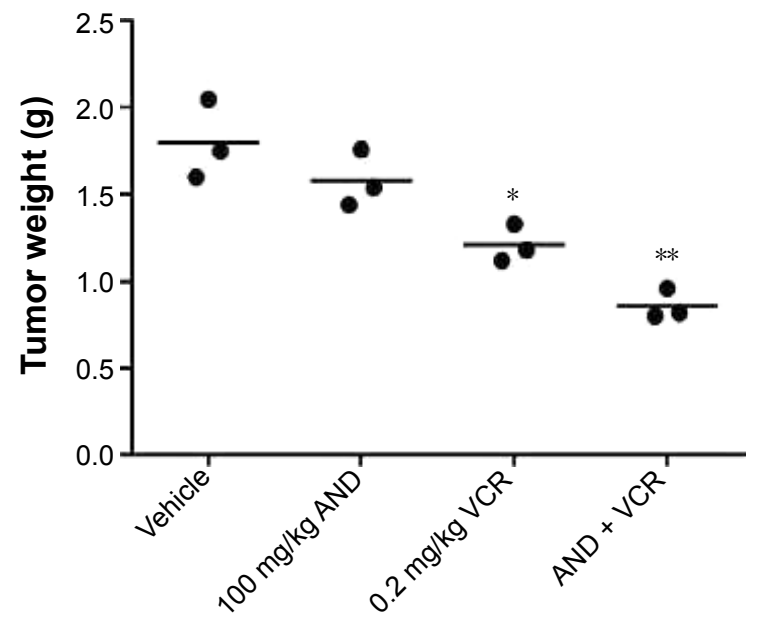

D

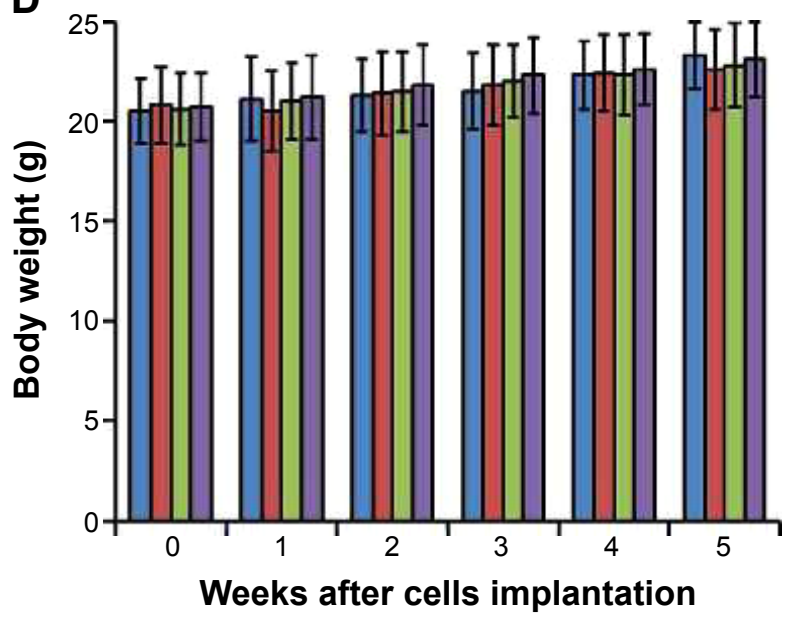

$\square$ Vehicle $\quad \square 100 \mathrm{mg} / \mathrm{kg} \mathrm{AND}$

$\square 0.2 \mathrm{mg} / \mathrm{kg}$ VCR $\square$ AND + VCR

Figure 6 VCR plus AND significantly inhibited SK-NEP-I xenograft tumor growth in vivo.

Notes: (A) $3 \times 10^{6}$ SK-NEP-I cells were subcutaneously injected in the right flank of the mice. Tumor volumes were monitored once a week. The mice were randomized to four groups and received vehicle (saline, oral, once daily), $100 \mathrm{mg} / \mathrm{kg}$ AND (oral, once daily), $0.2 \mathrm{mg} / \mathrm{kg}$ VCR (intraperitoneal, once weekly), and VCR plus AND. (B and C) After 4 weeks of treatment, all mice were sacrificed, and the tumors were weighted. (D) Body weight changes were monitored weekly during the process of efficacy study. Results are expressed as mean \pm standard deviation. $n=8, * P<0.05$, $* * p<0.01$, compared with vehicle group.

Abbreviations: AND, andrographolide; VCR, vincristine.

pathway, ${ }^{23}$ suppression of anti-apoptotic PI3K/AKT and ERK pathways, ${ }^{24}$ or activation of ERK-p53 apoptotic pathway. ${ }^{16}$ In order to reveal the underlying mechanisms when these two components were combined together, many biochemical methods were used. Evidence showed that autophagy was not involved in VCR- or/and AND-induced SK-NEP-1 cell death, because MDC did not change upon any treatment. The PI3K/AKT and p53 pathways are two most important regulatory pathways in cells where a fine balance between growth and response to stress helps in the homoeostasis of physiological conditions. ${ }^{25}$ They have been widely investigated in various kinds of cancer cells. Evidence showed that anaplasia was associated with significantly high levels of p53 expression. In the current study, data from Western blotting demonstrated that p-p53 was upregulated, and p-AKT level was downregulated when the cells were treated with VCR plus AND. AKT activator partly reversed apoptosis induced by VCR plus AND combination treatment in SK-NEP-1 cells further confirmed that PI3K-AKT-p53 pathway was involved. These results are similar to those of Tao et al's study, and TP53 and AKT are also confirmed to be two important upstream regulators in YM155-induced apoptotic pathway in SK-NEP-1 cells. ${ }^{21}$ Overexpression of the p53 tumor suppressor gene could be a marker of unfavorable WT tumors with poor prognosis and shorter survival period. ${ }^{26,27}$ However, the detailed mechanisms of p-p53 upregulated by 
VCR plus AND combination treatment are unclear, which requires further investigation.

\section{Conclusion}

The combination of VCR with AND has a strong synergistic antitumor effect on WT via PI3K-AKT-p53 signaling pathway, thereby representing a potential treatment for WT in the near future.

\section{Disclosure}

The authors report no conflicts of interest in this work.

\section{References}

1. Neville HL, Ritchey ML. Wilms' tumor. Overview of National Wilms' Tumor Study Group results. Urol Clin North Am. 2000;27(3):435-442.

2. Godzinski J. The current status of treatment of Wilms' tumor as per the SIOP trials. J Indian Assoc Pediatr Surg. 2015;20(1):16-20.

3. Metzger ML, Dome JS. Current therapy for Wilms' tumor. Oncologist. 2005;10(10):815-826.

4. Al-Hussain T, Ali A, Akhtar M. Wilms' tumor: an update. Adv Anat Pathol. 2014;21:166-173.

5. Ekenze SO, Agugua-Obianyo NE, Odetunde OA. The challenge of nephroblastoma in a developing country. Ann Oncol. 2006;17(10): $1598-1600$

6. Yao W, Li K, Xiao X, Gao J, Dong K, Lv Z. Outcomes of Wilms' tumor in eastern China: 10 years of experience at a single center. J Invest Surg. 2012;25(3):181-185.

7. Beckwith JB, Zuppan CE, Browning NG, Moksness J, Breslow NE. Histological analysis of aggressiveness and responsiveness in Wilms' tumor. Med Pediatr Oncol. 1996;27(5):422-428.

8. Green DM, Beckwith JB, Breslow NE, et al. Treatment of children with stages II to IV anaplastic Wilms' tumor: a report from the National Wilms' Tumor Study Group. J Clin Oncol. 1994;12(10):2126-2131.

9. Dome JS, Cotton CA, Perlman EJ, et al. Treatment of anaplastic histology Wilms' tumor: results from the fifth National Wilms' Tumor Study. J Clin Oncol. 2006;24(15):2352-2358.

10. Li J, Cheung HY, Zhang Z, Chan GK, Fong WF. Andrographolide induces cell cycle arrest at G2/M phase and cell death in HepG2 cells via alteration of reactive oxygen species. Eur J pharmacol. 2007; 568(1-3):31-44.

11. Cheung MT, Ramalingam R, Lau KK, Chiang WL, Chiu SK. Cell type-dependent effects of andrographolide on human cancer cell lines. Life Sci. 2012;91(15-16):751-760.

12. Chou TC. Theoretical basis, experimental design, and computerized simulation of synergism and antagonism in drug combination studies. Pharmacol Rev. 2006;58(3):621-681.
13. Liu Y, Yang Y, Ye YC, et al. Activation of ERK-p53 and ERK-mediated phosphorylation of Bcl-2 are involved in autophagic cell death induced by the c-Met inhibitor SU11274 in human lung cancer A549 cells. J Pharmacol Sci. 2012;118(14):423-432.

14. Philchenkov A. Caspases: potential targets for regulating cell death. J Cell Mol Med. 2004;8(4):432-444.

15. El-Khattouti A, Selimovic D, Haikel Y, Hassan M. Crosstalk between apoptosis and autophagy: molecular mechanisms and therapeutic strategies in cancer. J Cell Death. 2013;6:37-55.

16. Yang SH, Wang SM, Sye JP, et al. Andrographolide induces apoptosis of C6 glioma cells via the ERK-p53-caspase 7-PARP pathway. Biomed Res Int. 2014;2014:312847.

17. Pratheeshkumar P, Sheeja K, Kuttan G. Andrographolide induces apoptosis in B16F-10 melanoma cells by inhibiting NF- $\mathrm{\kappa B}-$ mediated bcl-2 activation and modulating $\mathrm{p} 53$-induced caspase-3 gene expression. Immunopharmacol Immunotoxicol. 2012;34(1):143-151.

18. Zhou J, Lu GD, Ong CS, Shen HM. Andrographolide sensitizes cancer cells to TRAIL-induced apoptosis via p53-mediated death receptor 4 up-regulation. Mol Cancer Ther. 2008;7(7):2170-2180.

19. Jo H, Mondal S, Tan D, et al. Small molecule-induced cytosolic activation of protein kinase AKT rescues ischemia-elicited neuronal death. Proc Natl Acad Sci U S A. 2012;109(26):10581-10586.

20. Yan FT, Zhi HL, Li XX, et al. Molecular mechanism of the cell death induced by the histone deacetylase pan inhibitor LBH589 (Panobinostat) in Wilms tumor cells. PLoS One. 2015;10(7):e0126566.

21. Tao YF, Lu J, Du XJ, et al. Survivin selective inhibitor YM155 induce apoptosis in SK-NEP-1 Wilms tumor cells. BMC Cancer. 2012;12:619.

22. Rajagopal S, Kumar RA, Deevi DS, Satyanarayana C, Rajagopalan R. Andrographolide, a potential cancer therapeutic agent isolated from Andrographis paniculata. J Exp Ther Oncol. 2003;3(3):147-158.

23. Ji L, Liu T, Liu J, Chen Y, Wang Z. Andrographolide inhibits human hepatoma-derived Hep3B cell growth through the activation of c-Jun N-terminal kinase. Planta Med. 2007;73(13):1397-1401.

24. Tsai HR, Yang LM, Tsai WJ, Chiou WF. Andrographolide acts through inhibition of ERK1/2 and AKT phosphorylation to suppress chemotactic migration. Eur J Pharmacol. 2004;498(1-3):45-52.

25. Abraham AG, O'Neill E. PI3K/AKT-mediated regulation of p53 in cancer. Biochem Soc Trans. 2014;42(4):798-803.

26. Jadali F, Sayadpour D, Rakhshan M, et al. Immunohistochemical detection of $\mathrm{p} 53$ protein expression as a prognostic factor in Wilms tumor. Iran J Kidney Dis. 2011;5(3):149-153.

27. Govender D, Harilal P, Hadley GP, Chetty R. p53 protein expression in nephroblastomas: a predictor of poor prognosis. Br J Cancer. 1998; 77:314-318.
Drug Design, Development and Therapy

\section{Publish your work in this journal}

Drug Design, Development and Therapy is an international, peerreviewed open-access journal that spans the spectrum of drug design and development through to clinical applications. Clinical outcomes, patient safety, and programs for the development and effective, safe, and sustained use of medicines are the features of the journal, which

\section{Dovepress}

has also been accepted for indexing on PubMed Central. The manuscript management system is completely online and includes a very quick and fair peer-review system, which is all easy to use. Visit http://www.dovepress.com/testimonials.php to read real quotes from published authors. 